\title{
Whatsapp: uma ferramenta pedagógica para o ensino de Química
}

Ingrid Maliszewski Paczkowski, IQ-UFRGS, ingridmaliszewski@ hotmail.com Camila Greff Passos, IQ-UFRGS, camila.passos@ufrgs.br

Resumo: Neste trabalho de natureza qualitativa, foi realizado um estudo com o objetivo de avaliar as potencialidades do uso do Whatsapp como ferramenta pedagógica no ensino de Química. Para tanto, foi criado um grupo no Whatsapp com alunos de uma turma do $2^{\circ}$ ano do Ensino Médio, de uma escola da rede pública estadual de Porto Alegre, como um espaço de educação não formal para fomentar o processo de ensinoaprendizagem. Conforme os registros no diário de campo e dos dados coletados com questionários, foi possível identificar que as atividades desenvolvidas com o uso do aplicativo possibilitaram a troca de vídeos, informações e discussões acerca da matéria estudada em sala de aula. Neste sentido, fortaleceram as interações entre alunos e professora na construção dos conhecimentos trabalhados.

Palavras-chave: Whatsapp, aprendizagem colaborativa, educação não formal.

\section{Whatsapp: a pedagogical tool for the teaching of Chemistry}

Abstract: In this qualitative work, a study was carried out to evaluate the potentialities of the use of Whatsapp as a pedagogical tool in the teaching of Chemistry. For that, a group of Whatsapp was created of a 2nd grade high school class of a public school located in Porto Alegre, as a non-formal education space to foster the teaching-learning process. According to the records in the field diary and the data collected with questionnaires, through the collected data, it was possible to identify that the activities developed with the application made possible the exchange of videos, information and discussions about the subject studied in the classroom. In this sense, they strengthened the interactions between students and teacher in the construction of the knowledge worked.

Keywords: Whatsapp, collaborative learning, non-formal education.

\section{Introdução}

Os principais benefícios elencados pelos professores na relação do ensino de Química com tecnologias são a possibilidade de aulas dinâmicas e interessantes aos alunos, a possibilidade do aumento das interações entre professor-aluno e o aumento da autonomia dos alunos nas atividades (Rosa \& Eichler, 2017). Adquiridos pela maioria dos estudantes, os aparelhos celulares merecem atenção no âmbito educacional, podendo ser aliados do processo de ensino-aprendizagem, uma vez que permitem o acesso à informação a qualquer hora e em qualquer lugar. Essa condição possibilita uma nova alternativa de ensino capaz de unir tecnologia e educação, também conhecida como Mobile Learning - aprendizagem com mobilidade (Moreira \& Simões, 2017). Os pontos positivos e negativos das redes sociais não mudam o fato de que essas ferramentas estão rapidamente se tornando populares, ganhando um lugar importante em nossas vidas e começando a ocupar seu lugar na educação (Raupp \& Eichler, 2012). Nos últimos anos, os aplicativos de mensagens instantâneas, que podem ser identificados como redes sociais baseadas em dispositivos móveis, começaram a se 
tornar populares. Embora existam muitos aplicativos de mensagens instantâneas que possam operar em dispositivos móveis, o Whatsapp é um dos mais utilizados (Statista, 2018).

Em estudos sobre o uso de diferentes plataformas de mensagens instantâneas na educação, foi demonstrado que esses aplicativos têm potencial para aumentar a aprendizagem (Smit \& Goede, 2012), para tornar os alunos mais ativos em seus estudos (Cifuentes \& Lents, 2010), para aumentar a interação entre alunos sobre temas pessoais, escolares e relacionados ao curso (Cifuentes \& Lents, 2010), para eliminar barreiras sociais (Doering et al., 2008) e para aumentar a motivação dos estudantes (Plana et al., 2013; Martins \& Gouveia, 2018). Devido a estes benefícios, que também são apoiados pelos estudos realizados utilizando especificamente o Whatsapp (Nguyen \& Fussell, 2015), nota-se que este pode ser uma ferramenta útil dentro do escopo de aprendizagem a qualquer hora e em qualquer lugar. O potencial das redes sociais, quando projetado de acordo com as necessidades da ciência e da informação, pode causar mudanças revolucionárias (Zaidieh, 2012). Por isso, sua influência no ambiente educacional pode ser considerada um tema pertinente para investigação.

O Whatsapp é definido como um aplicativo digital multimodal, multiplataforma, com alto grau de usabilidade (Plana et al., 2013). É possível verificar um alto grau de adesão popular a esse aplicativo, tornando possível a existência de novas experiências comunicativas entre os sujeitos. A ação comunicativa no aplicativo ocorre em um espaço democrático que permite diálogos espontâneos, como recurso didático se torna viável ao possibilitar a ação comunicativa entre os estudantes. Pois, gera conhecimento sem dissolver as relações entre professor e estudantes (Araújo \& Bottentuit, 2015).

A educação online se traduz em práticas educativas que podem ocorrer em ambientes formais e não formais, presenciais ou não, mediados por tecnologias móveis. A criação de um grupo de Whatsapp como ferramenta educacional pode servir como uma extensão da aula presencial. Conforme Naismith et al., (2009), para avaliar de modo pleno o potencial dos dispositivos móveis no processo de ensino- aprendizagem, é necessário observar além da sua utilização individual e compreender sua inserção nas práticas pedagógicas na sala de aula ou fora dela. A aprendizagem móvel ocorre dentro de uma situação específica criada a partir de atividades com objetivos, interesses e recursos disponíveis e, por isso, classifica-a em: livre, formal, digital, física e não formal (Frohberg et al., 2009).

Nesse sentido, este trabalho visa analisar as potencialidades da utilização do Whatsapp no ensino de Química para o favorecimento da interatividade dos estudantes entre si e com a professora durante as aulas e através da extensão da sala de aula como um espaço de educação não formal.

\section{Aprendizagem Colaborativa}

A aprendizagem colaborativa é uma abordagem para o ensino-aprendizagem, onde os alunos trabalham em grupos para resolver um problema, realizar uma tarefa ou criar um produto (Laal, 2012). A proposta da aprendizagem colaborativa é utilizar a interação entre os alunos e os temas de estudos, tornando o contato com o conteúdo mais dinâmico (Siqueira \& Alcântara, 2003). Dentro desta perspectiva, a aprendizagem móvel aliada com a aprendizagem colaborativa pode ser utilizada como meio para suscitar nos alunos maior interesse pela disciplina. Já que através do uso do Whatsapp, o grau de interatividade é imprescindível para estimular o aluno a aprender, motivando novas formas de relacionamento através da aprendizagem colaborativa durante o uso do aplicativo. Para fins educacionais, a utilização dessas ferramentas funciona como estratégia alternativa às abordagens tradicionais. 
Diversas pessoas pensam que o conhecimento é uma entidade que se transfere de uma cabeça para outra. A aprendizagem colaborativa, no entanto, parte da ideia de que o conhecimento é resultante de um consenso entre membros de uma comunidade de conhecimento, algo que as pessoas também constroem conversando, trabalhando juntas direta ou indiretamente (Torres et al., 2004). A criação de um grupo de Whatsapp encoraja a participação dos alunos em um processo de aprendizagem ativo e efetivo.

A aprendizagem colaborativa, por suas características próprias, representa um desdobramento teórico e metodológico das pedagogias e teorias existentes, propiciando uma forma de ensinar e aprender que supera o paradigma tradicional de ensino. Devido às inovações científicas e tecnológicas do mundo atual, ela apresenta-se como uma abordagem diferenciada para que os aprendizes possam ter condições de manusear a avalanche de informações às quais estão expostos, interpretando-as e transformando-as em conhecimentos socialmente relevantes (Torres et al., 2004). Assim, é possível construir o conhecimento socialmente, através da interação entre os alunos, reconhecendo o conhecimento prévio de cada estudante, sua experiência e seu entendimento de mundo. Ademais, o aluno pode desenvolver suas habilidades sociais e cognitivas de modo criativo e o professor é responsável por criar contextos e ambientes adequados, colocando os alunos em grupo, de maneira ordenada, e criando situações de aprendizagem. Dessa forma, todos os integrantes do grupo se tornam responsáveis por sua aprendizagem de maneira colaborativa e social ao invés de competitiva e isolada.

\section{Educação Formal e Não Formal}

A educação formal é aquela que se aprende na escola, com conteúdos demarcados, enquanto a não formal é aquela que se aprende 'no mundo da vida', via os processos de compartilhamento de experiências, principalmente em ações e espaços coletivos cotidianos (Gohn, 2006). É comum a educação formal ter como referência a escola enquanto que a educação não formal é utilizada para se referir a experiências distintas do ambiente escolar. As diferentes práticas educativas acontecem em lugares distintos, no ambiente familiar, escolar ou comunitário, e que cada prática possui um método de transmissão de ensinamento, seja ela formal ou não formal (Costa, 2011).

A educação formal pode ser resumida como aquela que encontramos no ensino escolar institucionalizado cronológico, gradual e hierarquicamente estruturado, sendo organizada sequencialmente e com foco na transmissão dos saberes para a manutenção da coesão do todo coletivo da sociedade (Bianconi \& Caruso, 2005).

A educação não formal pode ser definida como uma prática mais difusa, menos hierárquica e menos burocrática. Tanto a educação formal como a educação não formal são intencionais, contam com objetivos explícitos de aprendizagem ou formação e apresentam-se como processos educativamente diferenciados e específicos (Gadotti, 2005). A educação não formal possui múltiplos espaços: nos bairros, nas igrejas, nos sindicatos, nos espaços culturais, nas próprias escolas, nos espaços interativos escolares, com a comunidade educativa, entre outros.

Pode-se concluir que os conceitos de educação formal e não formal são flexíveis e estão relacionados aos contextos histórico e político envolvidos. Muitas vezes, o que era não formal pode passar a ser formal, assim como o que é formal em uma localidade pode não ser em outra. Assim, a educação não formal consegue satisfazer muitas lacunas deixadas pela educação formal. Neste trabalho, o uso do Whatsapp pode ser considerado uma forma de educação não formal, que vai além do ambiente da sala de aula, preenchendo as lacunas deixadas pela aula tradicional, em função do seu grande potencial de alcance, graças à sua liberdade e mobilidade para gerir suas práticas pedagógicas. 


\section{Metodologia}

Esta pesquisa de natureza qualitativa (Ludke \& Andre, 1986) foi realizada com 32 alunos de uma turma de $2^{\circ}$ ano do Ensino Médio de uma escola da rede pública estadual, localizada na cidade de Porto Alegre. A coleta de dados foi realizada através da aplicação de dois questionários adaptados de estudos anteriores (Ahada \& Lima, 2014, Moreira \& Simões, 2017). Um deles foi aplicado anterior à criação do grupo de Whatsapp, para diagnosticar as possibilidades do estudo e o outro ao término das aulas, para avaliar o recurso didático utilizado como complementar as atividades de sala de aula. Os registros do Diário de campo também foram considerados como fonte de dados.

Dos 32 alunos integrantes da turma, 28 concordaram em participar do grupo e se comprometeram a contribuir apresentando assuntos de interesse para serem discutidos no grupo e nas aulas de química. Os 28 estudantes e seus responsáveis legais assinaram um Termo de Consentimento Livre e Esclarecido declarando ciência do objetivo da pesquisa e autorizando a utilização dos dados coletados.

A análise de dados foi realizada de forma exploratória e interpretativa, para a triangulação dos dados proveniente das diferentes fontes e instrumentos, tais como, as produções escritas e orais dos alunos, os questionários propostos aos alunos e as informações que evidenciam a forma como os alunos vivenciaram a experiência registradas no Diário de Campo.

\section{$\underline{\text { Resultados e Discussão }}$}

Com os registros no diário de campo identificou-se que os estudantes apresentavam idade entre 15 e 17 anos. Faixa etária bastante equilibrada e adequada à seriação. Também foi realizado um levantamento sobre o que os alunos esperam da escola e dos professores. De acordo com os apontamentos obtidos pode-se constatar que os alunos possuem o desejo de aprender e consideram a escola o local adequado para isto, desde que os professores possibilitem atividades que eles possam participar de forma efetiva.

Após conhecer melhor a turma, os alunos foram convidados a participar de um grupo de Whatsapp com o objetivo de discutir conceitos, atualidades e conteúdos relacionados à Química. O convite foi feito através da aplicação de um questionário sobre tecnologias da informação, obtendo-se informações relevantes para o andamento do trabalho. Esse questionário foi aplicado aos 32 alunos, onde buscou-se saber se os alunos possuíam aparelho celular, se costumavam utilizar o Whatsapp, entre outras informações que são apresentadas no Gráfico 1.

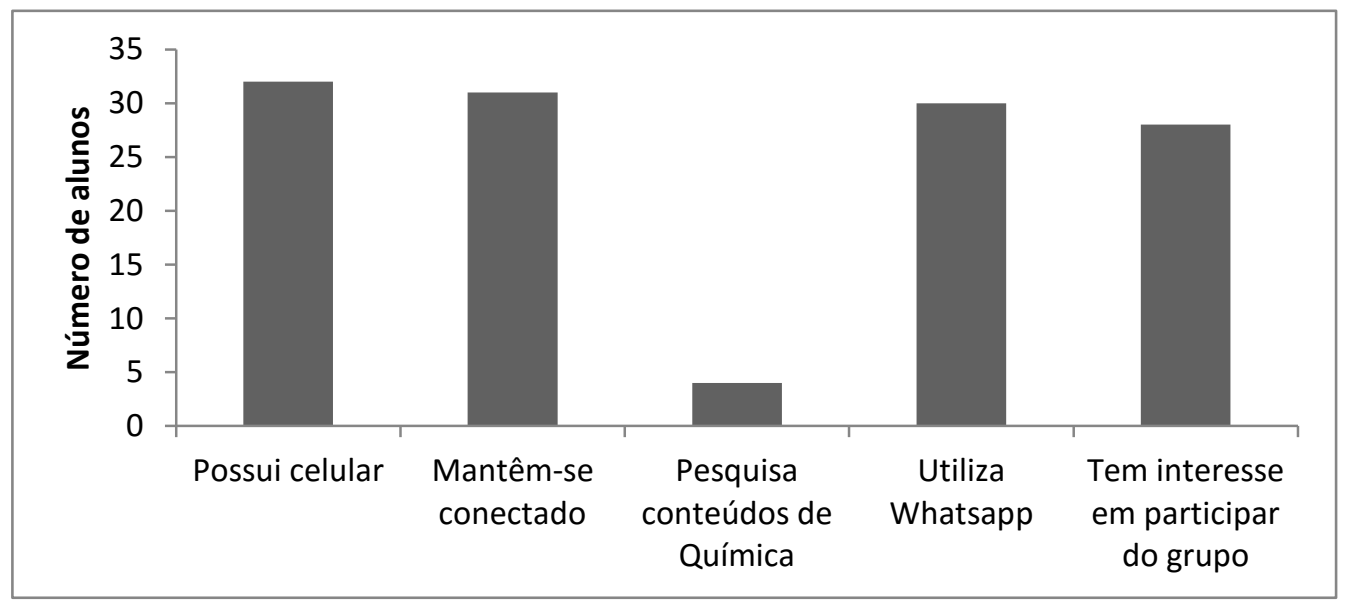


Gráfico 1. Respostas dos alunos ao questionário inicial sobre tecnologias da informação

Verificou-se que todos os alunos possuíam aparelho celular, o que tornou viável a criação do grupo de Whatsapp, visto que todos os alunos poderiam participar do mesmo. Além disso, a maioria dos alunos se mantinha conectado e possuía uma familiaridade com o aplicativo e tinha interesse em participar do grupo. Os alunos mostraram-se habituados com a utilização de aplicativos de mensagens instantâneas. Também foi importante saber que poucos alunos utilizam o celular para pesquisar conteúdos relacionados à Química, pois seria necessário fomentar o interesse dos alunos para essa finalidade. De acordo com Oliveira et al., (2014), para uma utilização eficaz do WhatsApp é necessário planejamento e organização, pois devido a dinâmica na troca de mensagens, a condução das interações entre os envolvidos pode se tornar difícil, e até negativa

Foi importante observar que a maioria dos alunos já utilizava o Whatsapp para organizar os trabalhos em grupo, então considerou-se que abordando temas interessantes e fomentando a participação dos alunos, provavelmente levaria ao êxito da atividade proposta. A aprendizagem colaborativa considera a ação mútua entre os sujeitos como fator que favorece o processo de ensino-aprendizagem, (Laal, 2012).

A partir destes dados, foi criado o grupo de Whatsapp, visando a interação e colaboração dos alunos em torno de temas de interesse coletivo relacionados à Química. Esse grupo serviu como uma extensão da sala de aula presencial.

Nas conversas do grupo criado foram identificadas características da escrita no Whatsapp: uso de emoticons e emojis, leitura dinâmica, uso de conteúdos digitais e linguagem informal. Além das características clássicas, a partir da captura de tela das conversas no grupo é possível identificar categorias que se encaixam nos contextos educativos dos conhecimentos de Química. Na Figura 1 é possível verificar o compartilhamento de vídeos relacionados ao conteúdo que estava sendo ministrado em sala de aula (reações endotérmicas e exotérmicas), mostrando que o Whatsapp funciona como uma extensão da sala de aula e que é possível direcionar o uso dos celulares para os objetivos educacionais.

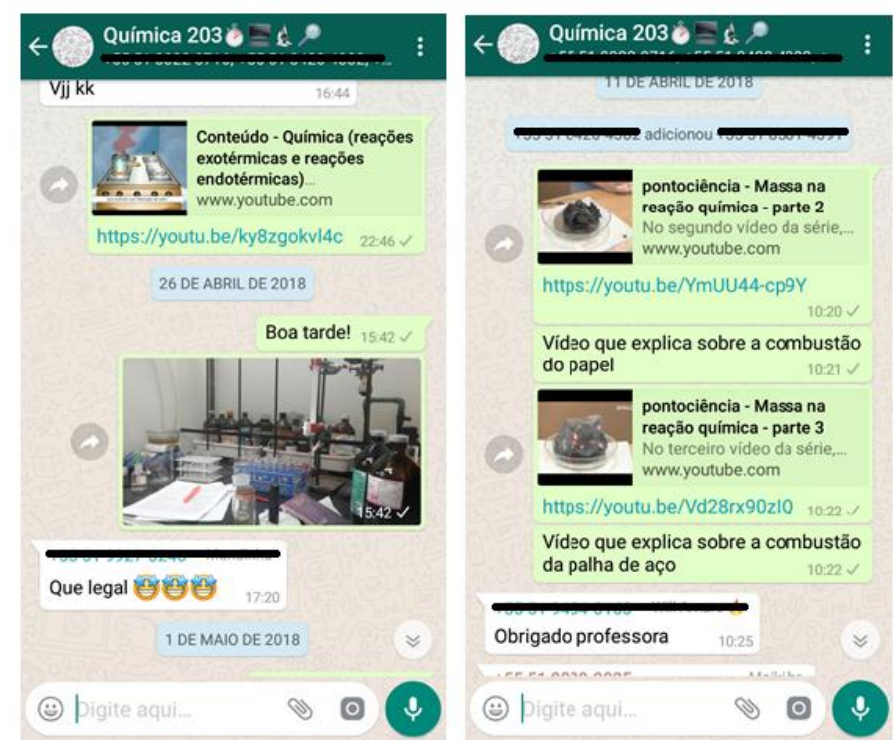

Figura 1. Utilização do Whatsapp para compartilhamento de vídeos com os alunos 
Por se tratar de uma escola sem muitos recursos para realização de experiências, o compartilhamento de vídeos em que eram realizadas demonstrações do conteúdo teórico estudado foi relevante para auxiliar os alunos a compreender melhor a relação entre os fenômenos demonstrados nos vídeos as teorias e representações discutidas em aula. Utilizando esses recursos, foi possível apresentar as diferentes situações que os conteúdos trabalhados durante as aulas podem ser relacionados, através dos diferentes fenômenos observados. Além disso, era possível citar as diferentes experiências em sala de aula e posteriormente utilizar o Whatsapp para compartilhar os vídeos com as demonstrações, do que anteriormente era apenas discutido em nível teórico. Foi possível verificar que ao utilizar essas ferramentas os alunos começaram a se interessar mais pela aula e pelo conteúdo. Em uma das atividades propostas, foi pedido para que os alunos pesquisassem na internet o que acontece com a massa dos produtos na combustão do papel e da palha de aço, para auxiliá-los na atividade, foram compartilhados vídeos via Whatsapp, conforme Figura 1.

A perspectiva da aprendizagem colaborativa visa fomentar a interação entre os alunos e os conteúdos, para assim suscitar nos alunos maior interesse pela disciplina (Siqueira \& Alcântara, 2003). Nesse sentido, entende-se que o uso do Whatsapp contribuiu para aumentar o grau de interatividade da turma com os conteúdos e com a professora, o que estimulou os alunos a aprenderem.

Inicialmente, foi possível observar que todos os estudantes visualizavam as mensagens, mas apenas alguns participaram ativamente do grupo, o restante participava de modo passivo. É possível considerar que ao ler as mensagens, os estudantes manifestaram o interesse em aprender e o grupo proporcionou uma oportunidade extra de construção do conhecimento, além da tradicional sala de aula. De acordo com Gadotti (2005), como a educação formal e não formal apresentam a intenção de ensinar, uma pode complementar a outra.

Com o objetivo de fomentar uma maior motivação por parte dos estudantes, aproveitou-se que estava na mídia o ataque de EUA, França e Reino Unido à Síria por utilização de armas químicas para promover uma discussão acerca do tema. Assim, foi possível incentivar alguns alunos a dialogar no Whatsapp e posteriormente discutir em sala de aula sobre o uso de armas químicas, podendo considerar que foi bastante produtivo trabalhar relacionando temas atuais com os conteúdos de química através do uso do Whatsapp, conforme Figura 2.
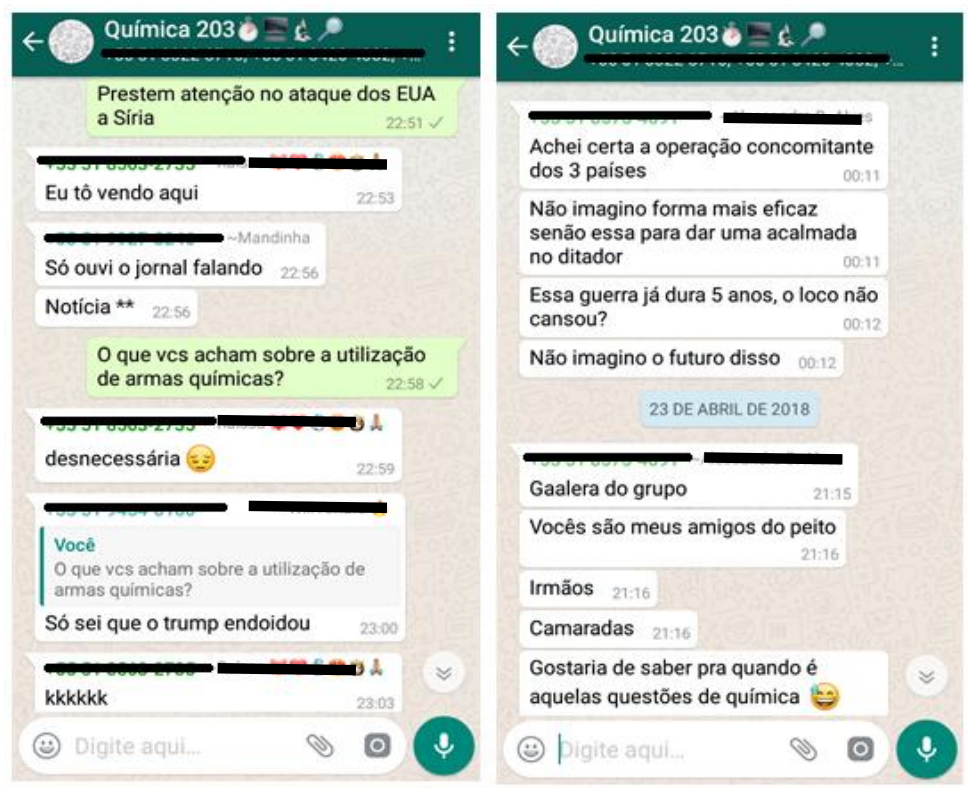
Também foi dada uma atividade que deveria ser feita no grupo para estimular, ainda mais, a interação dos alunos. A tarefa consistia em participar do evento Portas Abertas da UFRGS 2018 (UFRGS, 2019), postar no grupo alguma foto com o experimento de Química que mais gostou e entregar uma explicação sobre o fenômeno observado. A visita no Portas Abertas foi considerada como uma vivência do tipo não formal, pois tinha intencionalidade de ensino, mas em um espaço de compartilhamento social (Costa, 2011). Posteriormente, foi perguntado no grupo se os estudantes se interessaram pela atividade proposta. É possível notar, conforme Figura 3, que a tarefa foi bem sucedida e despertou o interesse dos alunos.

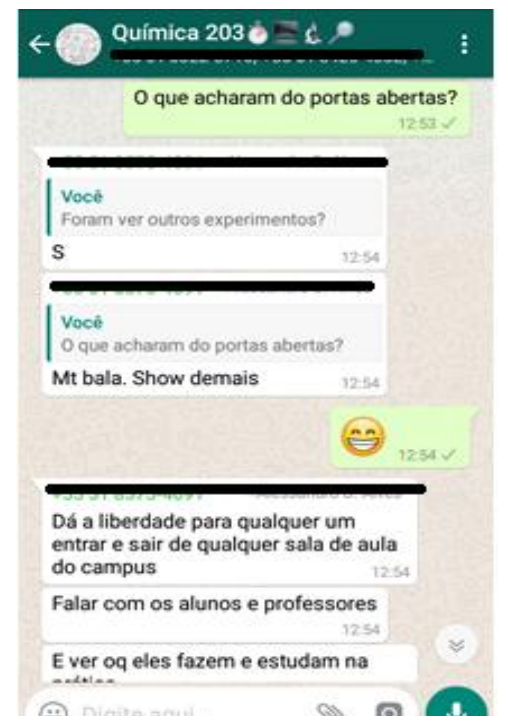

Figura 3. Realização de atividade avaliativa via Whatsapp

A última etapa da pesquisa, consistiu na aplicação de um questionário final com o objetivo de avaliar a opinião dos alunos sobre a utilização do Whatsapp como recurso de aprendizagem no ensino de Química. O questionário final foi composto de 8 perguntas com o objetivo de determinar a forma de participação, vantagens, desvantagens, importância, motivação, aprendizagem, relação social dos alunos em relação ao grupo. Ao analisar os resultados obtidos a partir da aplicação do questionário final, foi possível verificar que o Whatsapp pode ser considerado um recurso didático que favorece a interação entre alunos e professores na construção dos conhecimentos trabalhados e que motiva o estudo dos conteúdos de Química fora e em sala de aula, funcionando como uma extensão da sala de aula presencial.

Conforme Gohn (2006), a educação não formal é favorecida por processos de compartilhamento de experiências, principalmente em ações e espaços coletivos diferentes do ambiente escolar. Assim, pode mitigar lacunas do ensino formal.

Uma das perguntas abordadas neste questionário foi se os alunos se sentiram à vontade para participar do grupo. Analisando as respostas, foi possível verificar que todos se sentiram à vontade para participar do grupo, inclusive os mais tímidos. Neste âmbito a aprendizagem colaborativa foi favorecida com o uso do aplicativo. Para Torres et al. (2004), a utilização de ferramentas tecnológicas se apresentam como uma alternativa às abordagens tradicionais.

Todos os alunos apontaram vantagens na utilização do grupo de Whatsapp para discutir conceitos de Química, compartilhar vídeos e debater atualidades. A única desvantagem identificada foi que alguns alunos não possuíam plano de internet e necessitavam de acesso ao wifi para receber as mensagens. Por isso, as vezes recebiam 
as mensagens com um certo atraso. De acordo com os dados do diário de campo, foi observada uma mudança comportamental nos alunos após a criação do grupo de Whatsapp, pois eles passaram a conversar menos em sala de aula e debater mais assiduamente o conteúdo trabalhado. Além disso, muitos alunos relataram que os vídeos enviados foram importantes para uma melhor compreensão do conteúdo estudado em sala de aula. Verificou-se que todos os alunos consideraram o aplicativo uma nova possibilidade de aprendizado, as respostas dos alunos foram unanimes.

$\mathrm{O}$ grupo criado proporcionou o acesso à novas formas de ensinar e aprender. $\mathrm{O}$ planejamento e avaliação dessa ferramenta como recurso didático mostrou a importância do uso de tecnologias na educação, de forma convergente com relatos da literatura (Raupp \& Eichler, 2012; Plana et al., 2013; Martins \& Gouveia, 2018).

É pertinente salientar que o papel do professor é fundamental para que as informações facilmente acessadas sejam utilizadas para a construção de conhecimento socialmente relevante. Para isso, o professor tem que orientar a interação entre os alunos, a análise reflexiva dos dados e situações, para que os estudantes consigam desenvolver seus conhecimentos e habilidades de forma colaborativa (Torres et al., 2004).

\section{Conclusões}

Em função do alto grau de penetração social dos smartphones, foi viável seu uso como recurso didático na presente investigação. De acordo com o conjunto de dados analisados o uso do Whatsapp contribuiu para melhorar a qualidade das aulas, pois fomentou maior interatividade entre os estudantes e com a professora durante as aulas e através da extensão da sala de aula, como um espaço de educação não formal (Gohn, 2006).

Os dados obtidos estão de acordo com a literatura, considerando que o uso do Whatsapp contribuiu para o processo de aprendizagem colaborativa (Laal, 2012; Cifuentes \& Lents, 2010) e para aumentar a motivação dos estudantes (Plana et al., 2013; Martins \& Gouveia, 2018). Destaca-se que a turma participou das atividades, comentando sobre os vídeos, argumentando sobre as atualidades abordadas, postando fotos e discutindo sobre o evento Portas Abertas da UFRGS do qual participaram.

Após o término da atividade, o grupo de Whatsapp continuou ativo, mas as discussões sobre Química se encerraram quando se deixou de fomentar o interesse dos alunos para este fim. O Whatsapp passou a ser utilizado para discutir trabalhos em grupo de diversas disciplinas, confirmar os dias de provas e verificar quando os períodos seriam reduzidos. Isso comprovou que mesmo quando utilizamos recursos tecnológicos, a presença do professor é imprescindível para que o resultado final seja positivo (Torres et al., 2004).

As potencialidades identificadas neste estudo podem estar vinculadas aos estímulos fornecidos aos alunos ao inserir atualidades e temas do cotidiano relacionados à Química durante as discussões no grupo. Não é suficiente criar um grupo no Whatsapp para discutir o conteúdo dado em sala de aula, é necessário fomentar o interesse dos alunos. Assim, é possível aumentar a interação desses estudantes no grupo e melhorar a qualidade das aulas presenciais promovendo discussões acerca de vários assuntos relacionados à Química. 


\section{Referências}

AHADA, A. D.; LIMA, S. A. Convenience or Nuisance?: The 'WhatsApp' Dilemma. Procedia - Social and Behavioral Sciences, v. 155 p. 189 - 196, 2014.

ARAÚJO, P. C.; JUNIOR, J. B. B. O aplicativo de comunicação Whatsapp como estratégia no ensino de Filosofia. Temática, v. 11, n. 2, 2015.

BIANCONI, M. L.; CARUSO, F. Educação não-formal. Ciência e Cultura, v. 57, n. 4, p. 20-20, 2005.

CIFUENTES, O. E.; LENTS, N. H. Increasing student-teacher interactions at an urban commuter campus through instant messaging and online office hours. Electronic Journal of Science Education, v. 14, n. 1, p. 1-13, 2010.

COSTA, C. R. A importância da Educação não formal nas políticas públicas. Publicação periódica do Centro UNISAL, sob a coordenação do Programa de Mestrado em Educação Ano XIII-N ${ }^{\circ}$ 25-2 ${ }^{\circ}$ Semestre/2011 ISSN 15187039-CDU-37, p. 179, 2011.

DOERING, A.; LEWIS, C.; VELETSIANOS, G.; NICHOLS-BESEL, K. Preservice teachers' perceptions of instant messaging in two educational contexts. Journal of Computing in Teacher Education, V. 25, n. 1, p. 5-12, 2008.

FROHBERG, D.; GÖTH, C.; SCHWABE, G. Mobile learning projects-a critical analysis of the state of the art. Journal of computer assisted learning, v. 25, n. 4, p. 307-331, 2009.

GADOTTI, M. A questão da educação formal/não-formal. Sion: Institut Internacional des Droits de $1^{\circ}$ Enfant, p. 1-11, 2005.

GOHN, M. G. Educação não-formal, participação da sociedade civil e estruturas colegiadas nas escolas. Rio de Janeiro: Revista Ensaio-Avaliação e Políticas Públicas em Educação, v. 14, n. 50, p. 11-25, 2006.

LAAL, M.; LAAL, M. Collaborative learning: what is it?. Procedia-Social and Behavioral Sciences, v. 31, p. 491-495, 2012.

LUDKE, M.; ANDRÉ, M. E.D.A. Pesquisa em Educação: Abordagens Qualitativas. São Paulo: EPU, 1986.

MARTINS, Ernane Rosa; GOUVEIA, Luís Manuel Borges. O Uso do WhatsApp como Ferramenta de Apoio a Aprendizagem no Ensino Médio. RENOTE, v. 16, n. 2., 2018.

MOREIRA, M. L.; SIMÕES, A. S. M. O uso do whatsapp como ferramenta pedagógica no ensino de química. ACTIO, v. 2, n. 3, p. 21-43, outubro 2017.

NAISMITH, L. et al. Literature review in mobile technologies and learning. Futurelab Series (2004). University of Birmingham, Report, v. 11, 2009.

NGUYEN, D. T.; FUSSELL, S. R. Effects of conversational involvement cues on understanding and emotions in instant messaging conversations. Journal of Language \& Social Psychology, v. 35, n. 1, p. 28-55, fevereiro 2015.

OLIVEIRA, E. D. S. et al. Proposta de um modelo de cursos baseado em mobile learning: um experimento com professores e tutores no whatsapp. In: Anais do XI Congresso Brasileiro de Ensino Superior a Distância. Florianópolis/SC. 2014.p. 05-08.

PLANA, M. G. C.; ESCOFET, M. I. G.; FIGUERAS, I. T.; GIMENO, A.; APPEL, C.; HOPKINS, J. Improving learners' reading skills through instant short messages: A sample study using WhatsApp. 4th World-CALL Conference, Glasgow, julho 2013. Disponível

em: <https://www.researchgate.net/publication/255718202_Improving_learners'_reading_sk ills_thr ough_instant_short_messages_a_sample_study_using_WhatsApp>. Acesso em: 02 Jul. 2018. 
RAUPP, D.; EICHLER, M. L. A rede social Facebook e suas aplicações no ensino de química. RENOTE, v. 10, n. 1, 2012.

ROSA, M. P. A.; EICHLER, M. L. Brazilian teachers' beliefs about technologies in a training program in Portugal. Acta Scientiae, v. 19, n. 4, p. 679-692, julho 2017.

SIQUEIRA, L. M. M.; ALCÂNTARA, P. R. Modificando a atuação docente utilizando a colaboração. Revista Diálogo Educacional, v. 4, n. 8, p. 57-69, 2003.

SMIT, I.; GOEDE, R. WhatsApp with BlackBerry; Can messengers (BBM) be MXit?. Proceedings of the 14th annual conference on world wide web applications. Cape Town, South Africa: Cape Peninsula University of Technology, 2012. Disponível em: <http://hdl.handle.net/10394/13628>. Acesso em: 02 Jul. 2018.

STATISTA. Statistics and market data on mobile internet \& apps. The Statistics Portal, 2018. Disponível em: <https://www.statista.com/markets/424/topic/538/mobileinternet-apps/>. Acesso em: 02 Jul. 2018.

TORRES, P. L.; ALCANTARA, P.; IRALA, E. A. F. Grupos de consenso: uma proposta de aprendizagem colaborativa para o processo de ensinoaprendizagem. Revista diálogo educacional, v. 4, n. 13, p. 129-145, 2004.

UNIVERSIDADE FEDERAL DO RIO GRANDE DO SUL (UFRGS). Evento Portas Abertas UFRGS 2018. > https://www.ufrgs.br/portasabertas/programacao-2018/>. Acesso em 10 de Maio de 2019.

ZAIDIEH, A. J. Y. The use of social networking in education: challenges and opportunities. World of Computer Science and Information Technology Journal (WCSIT), v. 2, n. 1, p. 18-21, 2012. 